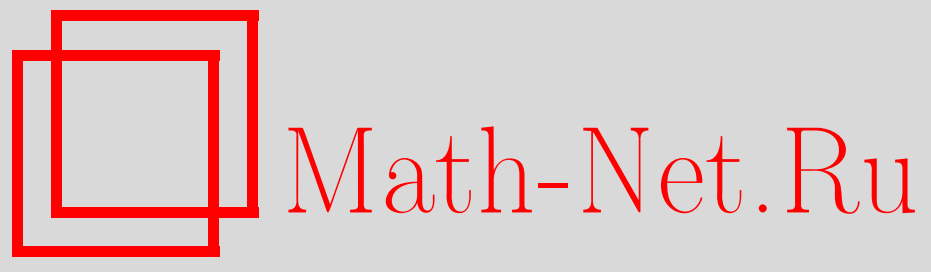

А. Г. Качуровский, Об энтропийном кирпиче автоморфизма пространства Лебега, Матем. заметки, 2006, том 80, выпуск 6, 943-945

DOI: https://doi.org/10.4213/mzm3371

Использование Общероссийского математического портала Math-Net.Ru подразумевает, что вы прочитали и согласны с пользовательским соглашением http://www . mathnet.ru/rus/agreement

Параметры загрузки:

IP: 3.82 .47 .9

26 апреля 2023 г., 15:46:20

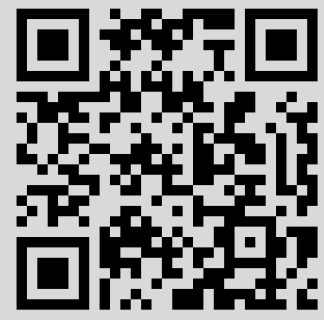




\section{ОБ ЭНТРОПИЙНОМ КИРПИЧЕ АВТОМОРФИЗМА ПРОСТРАНСТВА ЛЕБЕГА}

\section{А. Г. Качуровский}

Спектр и энтропия - два классических метрических инварианта автоморфизма пространства Лебега. Как хорошо известно - заведомо не полных, т.е. не дающих исчерпывающего решения проблемы метрического изоморфизма (нерешенной классической проблемы сопряженности в группе сохраняющих меру преобразований). Понятно, тем не менее, что полная система метрических инвариантов должна включать в себя и спектр, и энтропию. И поэтому имеет смысл поиск и изучение (хоть каких-нибудь) унифицирующих инвариантов. Одна из таких попыток и предлагается вниманию читателя.

Пусть $(\Omega, \lambda)$ - пространство Лебега, $T$ - его автоморфизм, $U_{T}$ - сопряженный с $T$ унитарный оператор, действующий на функциях $f$ из (сепарабельного комплексного гильбертова) пространства $L_{2}(\Omega)$ по формуле $U_{T} f=f \circ T$. И пусть

$$
\rho_{1}\left(U_{T}\right) \geqslant \rho_{2}\left(U_{T}\right) \geqslant \cdots
$$

- спектральная последовательность оператора $U_{T}$, т.е. последовательность типов Хеллингера (классов эквивалентности по отношению взаимной абсолютной непрерывности) борелевских мер на единичной окружности, являющаяся хорошо известной в анализе системой обычных унитарных инвариантов оператора $U_{T}$ (дающих полное решение проблемы сопряженности в группе унитарных операторов сепарабельного гильбертова пространства); удобно считать эту последовательность бесконечной - дополняя, если она обрывается на конечном шаге, нулями [1], [2; § 2], [3; приложение 2].

Для каждого разложения $\bigoplus U_{k}$ оператора $U_{T}$ в ортогональную сумму циклических частей (со спектральными типами $\rho\left(U_{k}\right)=\rho_{k}\left(U_{T}\right)$, удовлетворяющими соотношению $(1)$ рассмотрим разложение пространства $L_{2}(\Omega)$ в ортогональную сумму $\bigoplus H_{k}$ соответствующих циклических подпространств. Каждое такое $H_{k}$ порождает минимальную $\sigma$-алгебру на $\Omega$, относительно которой измеримы все функции из $H_{k}$; обозначим соответствующее ей измеримое разбиение пространства $\Omega$ через $\xi_{k}$. Каждому такому разбиению $\xi_{k}$ соответствует фактор-автоморфизм $T_{\xi_{k}}$ фактор-пространства Лебега $\Omega / \xi_{k} ;$ пусть $h_{k}$ - энтропия этого автоморфизма.

Таким образом, для каждого рассматриваемого разложения $\bigoplus U_{k}$ мы получаем последовательность пар $\left(\rho_{k}\left(U_{T}\right), h_{k}\right), k=1,2, \ldots$. Как хорошо известно, спектральная последовательность (1) от выбора конкретного разложения $\bigoplus U_{k}$ оператора $U_{T}$ не зависит (и является метрическим инвариантом автоморфизма $T$ ). Чего нельзя, вообще говоря, утверждать о последовательности $h_{k}, k=1,2, \ldots$ (и, соответственно, о последовательности пар $\left.\left(\rho_{k}\left(U_{T}\right), h_{k}\right)\right)$. Поэтому имеет смысл рассмотреть множество всех последовательностей $\left(\rho_{k}\left(U_{T}\right), h_{k}\right)$ - по всем возможным рассматриваемым разложениям $\bigoplus U_{k}$ оператора $U_{T}$; назовем это множество энтропийным кирпичом автоморфизма $T$.

Теорема. Энтропийный кирпич - метрический инвариант автоморфизма пространства Лебега, содержащий полную информачию о спектре и энтропии.

ДокАзАТЕЛЬство. То, что рассматриваемый кирпич является метрическим инвариантом, сразу следует из метрической инвариантности обычных спектра и энтропии. Вся информация о спектральной последовательности (1) автоморфизма непосредственно заложена в структуре его кирпича. Остается понять, как по кирпичу вычисляется обычная

Работа выполнена при частичной поддержке Российского фонда фундаментальных исследований, грант № 03-01-00403.

(C) А. Г. КАчуровский, 2006 
энтропия автоморфизма. Естественно предположить, что она получается просто как супремум его энтропийных координат. Докажем это.

Пусть $Z_{0}$ - множество всех конечных измеримых разбиений пространства Лебега $(\Omega, \lambda)$. Для каждого $\xi \in Z_{0}$ (с элементами разбиения $\left.C_{1}, \ldots, C_{m}\right)$ положим, как обычно [2], [3; гл. $10, \S 6]$,

$$
\begin{gathered}
H(\xi)=-\sum_{i=1}^{m} \lambda\left(C_{i}\right) \ln \left(\lambda\left(C_{i}\right)\right), \\
h(T, \xi)=\lim _{n \rightarrow \infty} \frac{1}{n} H\left(\bigvee_{k=0}^{n-1} T^{k} \xi\right), \quad h(T)=\sup _{\xi \in Z_{0}} h(T, \xi) .
\end{gathered}
$$

Для этих же $\xi \in Z_{0}$ рассмотрим

$$
\xi_{T}=\bigvee_{k=-\infty}^{\infty} T^{k} \xi
$$

- вполне инвариантное (т.е. и $T$-инвариантное, и $T^{-1}$-инвариантное) измеримое разбиение пространства $\Omega$, порождающее соответствующий ему фактор-автоморфизм $T_{\xi_{T}}$, действующий на фактор-пространстве $\Omega / \xi_{T}$, тоже являющемся пространством Лебега $[2$; $\S 1]$. Заметим, что

$$
h(T, \xi)=h\left(T_{\xi_{T}}, \xi\right) .
$$

Так как разбиение $\xi$ - двусторонняя образующая фактор-автоморфизма $T_{\xi_{T}}$ (т.е. $\xi_{T_{\xi_{T}}}$ дает разбиение фактор-пространства $\Omega / \xi_{T}$ на атомы), то [2; § 9], [3; глава $\left.10, \S 6\right]$

$$
h\left(T_{\xi_{T}}, \xi\right)=h\left(T_{\xi_{T}}\right)
$$

Поэтому

$$
h(T)=\sup _{\xi \in Z_{0}} h(T, \xi)=\sup _{\xi \in Z_{0}} h\left(T_{\xi_{T}}, \xi\right)=\sup _{\xi \in Z_{0}} h\left(T_{\xi_{T}}\right) .
$$

Остается понять, что для каждого рассматриваемого $\xi$ соответствующая измеримому разбиению $\xi_{T} \sigma$-алгебра является (наименьшей) $\sigma$-алгеброй, относительно которой измеримы все функции циклического подпространства, порожденного некоторой ступенчатой функцией. Для этого достаточно взять любую ступенчатую функцию $f$, постоянную на элементах (конечного измеримого) разбиения $\xi$ и принимающую различные значения на разных его элементах, и рассмотреть порожденное ею циклическое подпространство оператора $U_{T}$. Тогда $\sigma$-алгебра, порождаемая линейными комбинациями функций $U_{T}^{k} f$, $-\infty<k<\infty$ (т.е. наименьшая $\sigma$-алгебра, относительно которой все эти линейные комбинации измеримы), совпадает с $\sigma$-алгеброй, соответствующей разбиению $\xi_{T}$. Остается заметить, что замыкание множества этих линейных комбинаций в $L_{2}(\Omega)$ (т.е. порождаемое функцией $f$ циклическое подпространство оператора $\left.U_{T}\right)$ указанной $\sigma$-алгебры не расширяет (т.е. все функции из этого замыкания все еще измеримы относительно нее), что сразу следует, например, из полноты меры Лебега. Теорема доказана.

ЗАмечАниЕ 1. Посмотрим, как могут выглядеть энтропийные кирпичи различных классов автоморфизмов.

Если энтропия автоморфизма равна нулю, то и любой его фактор-автоморфизм имеет нулевую энтропию. В этом случае все энтропийные координаты кирпича просто зануляются и он вырождается в обычную спектральную последовательность (1).

Пусть теперь $T$ - эргодический автоморфизм с положительной энтропией. Как известно $[2 ; \S 11]$, в этом случае существует (вполне инвариантное измеримое) разбиение Пинскера $\pi(T)$, которое есть наименьшая верхняя грань всех разбиений $\xi$ с $h(T, \xi)=0$ (соответственно фактор-автоморфизм $T_{\pi(T)}$ является наибольшим из имеющих нулевую энтропию фактор-автоморфизмов автоморфизма $T)$. И если обозначить через $L_{2}(\Omega) \ominus$ 
$L_{2}(\Omega, \pi)$ ортогональное дополнение в $L_{2}(\Omega)$ к подпространству $L_{2}(\Omega, \pi)$ функций, постоянных на элементах разбиения $\pi(T)$, то на этом инвариантном подпространстве оператор $U_{T}$ имеет $[2 ; \S 14]$ счетнократный лебеговский спектр (т.е. спектральный тип Хеллингера циклического подпространства, порожденного любой функцией из $L_{2}(\Omega) \ominus L_{2}(\Omega, \pi)$, совпадает со спектральным типом меры Лебега на окружности). Понятно, что для функций из $L_{2}(\Omega, \pi)$ все соответствующие им энтропийные координаты - нули. Энтропийные координаты функций из $L_{2}(\Omega) \ominus L_{2}(\Omega, \pi)$ радуют большим разнообразием. Как известно [4], в этом случае у $T$ есть (изоморфные автоморфизмам Бернулли) факторавтоморфизмы с любой меньшей $h(T)$ энтропией (с соответствующими конечными двусторонними образующими разбиениями).

ЗАмечАниЕ 2. Можно, по аналогии с описанным выше энтропийным кирпичом, рассматривать и другие аналогичные ему метрические инварианты автоморфизма.

Например, можно формировать кирпич не парами $\left(\rho_{k}\left(U_{T}\right), h_{k}\right)$, а тройками $\left(\rho_{k}\left(U_{T}\right)\right.$, $\left.h_{k}, \bar{\xi}_{k}\right)$, для метрического типа $\bar{\xi}_{k}$ измеримого разбиения $\xi_{k}$ пространства $\Omega$ (порождаемого соответствующим циклическим подпространством $H_{k}$, т.е. условием измеримости всех входящих в него функций - см. выше процедуру формирования обычного кирпича); здесь метрический тип разбиения - это его класс эквивалентности по отношению изоморфизма разбиений. Понятно, что такой кирпич, тоже являясь метрическим инвариантом автоморфизма, заведомо содержит всю информацию о рассмотренном в теореме энтропийном кирпиче. Можно, конечно, рассматривать также и кирпич, формируемый просто парами $\left(\rho_{k}\left(U_{T}\right), \overline{\xi_{k}}\right)$.

И, наконец, можно рассматривать аналоги всех описанных выше кирпичей, строя циклические подпространства только по ступенчатым функциям (с конечным числом ступенек с измеримыми основаниями): так как множество таких ступенчатых функций плотно в $L_{2}(\Omega)$, то стандартная процедура [1] построения спектральной последовательности (1) оператора $U_{T}$ позволяет это сделать.

\section{СПИСОК ЦИТИРОВАННОЙ ЛИТЕРАТУРЫ}

[1] А.И. Плеснер, Спектральная теория линейных операторов, Наука, М., 1965. [2] В. А. Рохлин, УМН, 22:5 (1967), 3-56. [3] И. П. Корнфельд, Я. Г. Синай, С. В. Фомин, Эргодическая теория, Наука, М., 1980. [4] Я. Г. Синай, Матем. сб., 63:1 (1964), 23-42.

\section{А. Г. Качуровский}

Институт математики им. С. Л. Соболева

Поступило

Сибирского отделения РАН, г. Новосибирск

E-mail: agk@math.nsc.ru 